\title{
Vertical wage differences in hierarchically structured firms
}

\author{
René van den Brink
}

Received: 10 October 2005 / Accepted: 23 February 2007 / Published online: 4 April 2007

(C) Springer-Verlag 2007

\begin{abstract}
In this paper, we present a cooperative model of a hierarchically structured firm to study wage differences between different levels in such a firm. We consider a class of wage functions that are based on marginal contributions to production. It turns out that the wage of a manager is always at least as high as the wage of its subordinates. On the other hand, the wage of a manager never exceeds the sum of the wages of its direct subordinates. These bounds are sharp in the sense that we can characterize for which production processes they are reached. For the class of constant elasticity of substitution (CES) production functions this implies that the wage differences are maximal for linear production functions, and they are minimal for Cobb-Douglas production functions.
\end{abstract}

\section{Introduction}

In most hierarchically structured firms not all wages are equal. Within a particular hierarchical level, there can be wage differences because of differences in the importance of the tasks done or the scarcity of the labor inputs that are able to perform the different tasks. Besides these horizontal wage differences within levels, there are also vertical wage differences between different levels. This paper studies this second kind of wage differences. A hierarchically structured firm is represented by a hierarchical production game which consists of a set of participants or employees (workers and managers), a hierarchical firm structure and a cooperative production game.

R. van den Brink $(\varangle)$

Department of Econometrics and Tinbergen Institute, Free University, De Boelelaan 1105,

$1081 \mathrm{HV}$, Amsterdam, The Netherlands

e-mail: jrbrink@feweb.vu.nl 
Following the seminal paper of Alchian and Demsetz (1972), we assume that production is in principle a collective effort in which teams of agents access the productive asset and generate a collective production output value (see also, Ichiishi 1993). Therefore, we model production as a cooperative game. The firm structure is assumed to have a tree structure with a unique position at the top. This implies that there are positions that have no subordinates, i.e. the leafs of the tree. We assume that the production process is carried out by the employees on these "bottom" positions, as also done in other models of hierarchical firms such as, e.g., the seminal papers by Simon (1957), Williamson (1967), Calvo and Wellisz (1979), Keren and Levhari (1979, 1983), and Radner (1992). We call these employees the workers of the firm. They form the set of players in the cooperative production game. The employees in higher levels are managers or coordinators who organize and coordinate the production process. The coordination task of managers or coordinators can have various forms, such as information processing, monitoring, and decentralized decision making. We do not explicitly specify the role of managers in this paper, but assume that their role in the production process gives them a higher position in the firm hierarchy.

This paper introduces a cooperative wage system that remunerates the employees in a firm by assuming that every employee needs approval (or instructions) of its direct superior in order to be active in the production process. ${ }^{1}$ The wages of the workers and the managers on the different levels of the firm structure are determined by applying the cooperative concept of marginal contribution. The marginal contribution of an employee to a set of employees is the decrease in production output value when this employee stops being active, assuming that the given set of employees is active. This implies that the wages of firm employees not only depend on total production that can be generated by the fully employed firm, but also depend on the production capabilities of subsets of employees, i.e. on the production that can be generated by the firm if some positions are vacant. Taking a weighted average over all marginal contributions of a particular player yields its weighted average marginal contribution. Since we require budget neutrality of the wage function, i.e. the sum of all wages is exactly equal to the production output value of the fully employed firm, we normalize the weighted average marginal contributions so that we obtain a budget neutral wage function that distributes the total production output value proportional to the weighted average marginal contributions of the employees. Our main results show that according to these wage functions, the wage of a manager always is at least as high as the wage of every employee that is subordinate to this manager. On the other hand, the wage of a manager never exceeds the sum of the wages of its direct subordinates. It is also shown that these bounds are sharp in the sense that there are production technologies for which these bounds are reached. A manager earns the same wage as one of its subordinates if this subordinate is indispensable for the manager, i.e. the workers that are coordinated by the manager but not by this subordinate need

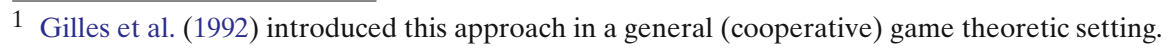


workers that are coordinated by the subordinate in order to be productive. A manager earns the sum of the wages of its direct subordinates if all its subordinate workers are so-called dummy workers, i.e. there are no synergies when a subordinate worker cooperates with other workers.

In order to focus on the hierarchical aspect of vertical wage differences, we also consider the special case in which all workers are identical in the production process, and all managers within one level take similar positions in the hierarchical firm structure in the sense that the firm has constant span of control, i.e. every manager has the same number of direct subordinates. In that case the ratio between the wage of a manager and each of its direct subordinates lies between one and the span of control. These bounds are often assumed and supported in the literature, see e.g., Simon (1957), Williamson (1967), Calvo and Wellisz (1978, 1979), Carlson (1982), and Radner (1992). For firms producing according to a constant elasticity of substitution (CES) production technology, this implies that the ratio between the wage of a manager and each of its direct subordinates reaches the upper bound (equal to the span of control) for linear production technologies with substitutable labor inputs, while it reaches the lower bound (equal to one) for Cobb-Douglas production technologies with indispensable labor inputs.

The paper is organized as follows. Section 2 describes the model of a hierarchically structured firm. In Sect. 3, we introduce the class of weighted average marginal contributions (WAMC) wage functions. In Sect. 4, we state the main results on comparing wages between different hierarchical levels within a firm. In Sect. 5, we state some additional properties and characterize the WAMCwage functions. Section 6 illustrates the model using the Shapley wage function [based on the Shapley value for cooperative games, see Shapley (1953)] in a particular CES production firm. Section 7 contains concluding remarks and relations to the literature. Finally, all proofs are relegated to an appendix.

\section{Hierarchically structured firms}

A hierarchically structured firm is modelled as a triple $(N, S, v)$, where the finite set $N$ is the set of employees in the firm, $S$ represents the hierarchical structure of the firm, and $v$ is a production game describing the potential production possibilities of the workers in the firm.

\subsection{The firm structure}

The firm structure $S: N \rightarrow 2^{N}$ with $i \notin S(i)$ is a mapping that assigns to every employee $i \in N$ in a firm those employees that are directly subordinate to $i$. These direct subordinates in the set $S(i) \subset N$ are called the successors ${ }^{2}$ of $i$. On the other hand, the employees in the set $S^{-1}(i):=\{j \in N \mid i \in S(j)\}$ are

\footnotetext{
2 Note that the mapping $S$ describes a directed graph $\{(i, j) \in N \times N \mid i \in N, j \in S(i)\}$.
} 
$i$ 's direct superiors and are referred to as the predecessors of $i$. By $\widehat{S}$ we denote the transitive closure of the firm structure $S$, i.e., $j \in \widehat{S}(i)$, if and only if there exists a sequence of employees $\left(h_{1}, \ldots, h_{t}\right)$ such that $h_{1}=i, h_{k+1} \in S\left(h_{k}\right)$, for all $1 \leq k \leq t-1$, and $h_{t}=j$. We refer to the employees in $\widehat{S}(i)$ as the subordinates of employee $i$. Similarly, we refer to the employees in the set $\widehat{S}^{-1}(i)=\{j \in N \mid i \in \widehat{S}(j)\}$ as the superiors of employee $i$. As usual in the firm literature, we assume that the firm has a tree structure, i.e., there is a unique root or top-position $i_{0}$ having no predecessors and being a superior to all other positions, while all other positions have exactly one predecessor and are not subordinate to themselves. Formally,

- there is exactly one employee $i_{0} \in N$ such that $S^{-1}\left(i_{0}\right)=\emptyset$ and $\widehat{S}\left(i_{0}\right)=$ $N \backslash\left\{i_{0}\right\}$

- for every $i \in N \backslash\left\{i_{0}\right\}$ it holds that $\left|S^{-1}(i)\right|=1$ and $i \notin \widehat{S}(i)$,

where $|E|$ denotes the cardinality of $E \subseteq N$. By $S$ having a tree structure it follows that there always exist employees that have no successors. These employees are referred to as the workers in $S$ and the set of workers in $S$ is denoted by $W_{S}=\{i \in N \mid S(i)=\emptyset\}$. We assume that these workers operate the production process in the firm. The other employees are the managers or coordinators who do not actively produce but who coordinate the production process. The set of managers in $S$ is denoted by $M_{S}=N \backslash W_{S}$. For a manager $i \in M_{S}$, the set $\bar{S}(i)=\widehat{S}(i) \cap W_{S}$ is the set of workers that are (directly or indirectly) subordinate to $i$. For notational convenience, we denote $\bar{S}(i)=\{i\}$ for every worker $i \in W_{S}$.

Example 2.1 Consider the set of employees $N=\{1, \ldots, 8\}$ and firm structure $S$ given by $S(1)=S(2)=S(3)=S(4)=\emptyset, S(5)=\{1,2\}, S(6)=\{3,4\}, S(7)=$ $\{6\}, S(8)=\{5,7\}$ (see Fig. 1$)$.

The set of workers in the firm structure $S$ is $W_{S}=\{1,2,3,4\}$. The set of managers in $S$ is the set $M_{S}=\{5,6,7,8\}$. The set of all (direct and indirect) subordinates of manager 7 is the set $\widehat{S}(7)=\{3,4,6\}$. The workers subordinate to manager 7 are the ones in the set $\bar{S}(7)=\{3,4\}$.

\subsection{The production game}

Since the production process is carried out by the workers in $W_{S}$, its power set is the domain of the (cooperative) production game $v: 2^{W_{S}} \rightarrow \mathbb{R}_{+}$. The value $v(E) \in \mathbb{R}_{+}$is the nonnegative production output value that can be generated

Fig. 1 The firm structure $S$ of Example 2.1

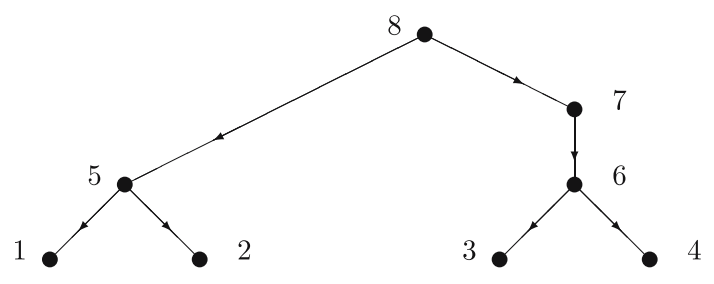


if exactly the workers in $E \subseteq W_{S}$ are active in the production process. ${ }^{3}$ So, it is assumed that every worker $i \in W_{S}$ can choose either to provide all its labor effort or to provide nothing at all. Alternatively, we can think that firm positions can be occupied by employees or be vacant.

We refer to a triple $(N, S, v)$ as a hierarchical production game. Throughout the paper we assume that the production game satisfies the zero input condition meaning that nothing can be produced if there are no workers to operate the production process, i.e. $v(\emptyset)=0$. We also require the productive fully employed firm condition meaning that a fully employed firm produces a positive production output value, i.e. $v\left(W_{S}\right)>0$. Other properties that are sometimes required are the following. A production game $v$ is monotone if $v(E) \leq v(F)$ whenever $E \subseteq F$. A production technology that exhibits complementarities with respect to the labor inputs is represented by a supermodular production game ${ }^{4}$ meaning that $v(E)+v(F) \leq v(E \cup F)+v(E \cap F)$ for all $E, F \subseteq W_{S}$.

\section{Wage functions based on marginal contributions}

The pay system that is used in rewarding the various positions in the firm is given by a wage function $\varphi$ that assigns a nonnegative wage $\varphi_{i}(N, S, v)$ to every employee $i \in N$ in the corresponding hierarchical production game $(N, S, v)$. A basic requirement for wage functions is that they always exactly distribute the total production output value over the employees, i.e. $\sum_{i \in N} \varphi_{i}(N, S, v)=v\left(W_{S}\right)$ for all $(N, S, v)$. This property is referred to as budget neutrality. ${ }^{5}$

We consider a class of wage functions according to which the wages of employees are determined by some weighted average of their marginal contributions to production. We do this in two steps. First, we need to extend the domain of the production game $v$ to make it a game on all employees in $N$. We do this by adding all managers in $M_{S}$ as null players to the production game $v$, i.e. as players who do not contribute to any coalition. So, we define the game $\bar{v}: 2^{N} \rightarrow \mathbb{R}_{+}$by $\bar{v}(E)=v\left(E \cap W_{S}\right)$ for all $E \subseteq N$. To acknowledge their management role in the production process, we follow Gilles et al. (1992), and define the modified production game $r_{S, v}: 2^{N} \rightarrow \mathbb{R}_{+}$by $r_{S, v}(E)=\bar{v}(\operatorname{sov}(E))$, where $\operatorname{sov}(E)=\left\{i \in E \mid \widehat{S}^{-1}(i) \subset E\right\}$ is the sovereign part of $E$, i.e. it is the set of those employees in $E$ whose superiors all belong to $E$. This reflects the idea that in order to activate a worker, all its superior managers should be active or

\footnotetext{
3 Assuming labor cost to be the only cost, and the firm to be a perfect competitor on its output market, this production output value is the value added that is generated by these labor inputs, i.e. the output multiplied by the competitive output price.

${ }^{4}$ In the game theory literature this property is usually referred to as convexity. Milgrom and Roberts (1994) stress the importance of coordination for supermodular production technologies.

5 In game theory terminology this property is called efficiency. The model can be easily adapted to include profits. In that case budget neutrality states that the sum of all wages and profit equals the total production output value. Since we concentrate on the internal wage distribution, we ignore profit in this paper.
} 
approve of the action taken by the worker. (Note that, equivalently, we could say that every worker and manager needs approval of its predecessor.)

The marginal contribution $M_{i}^{E}(N, S, v)$ of employee $i \in N$ to the set of employees $E \subseteq N$ in the hierarchical production game $(N, S, v)$ is the decrease in production output value according to the modified production game $r_{S, v}$ when employee $i$ stops being active, given that $E$ is the set of active employees, i.e. $M_{i}^{E}(N, S, v)=r_{S, v}(E)-r_{S, v}(E \backslash\{i\})$.

Next, we assign to every employee $i \in N$ its weighted average marginal contribution according to some positive weight system $\omega=\left(\omega_{1}, \ldots, \omega_{|N|}\right)$ with $\omega_{k}>0, k \in\{1, \ldots,|N|\}$. These weights $\omega_{k}$ express the importance of coalitions of different size $k$ in determining wages. ${ }^{6}$ The corresponding weighted average marginal contribution ${ }^{7}$ then is given by

$$
\begin{aligned}
m_{i}^{\omega}(N, S, v) & =\sum_{\substack{E \subseteq N \\
i \in E}} \omega_{|E|} M_{i}^{E}(N, S, v)=\sum_{\substack{E \subseteq N \\
i \in E}} \omega_{|E|}\left(r_{S, v}(E)-r_{S, v}(E \backslash\{i\})\right) \\
& =\sum_{\substack{E \subseteq N \\
i \in E}} \omega_{|E|}(\bar{v}(\operatorname{sov}(E))-\bar{v}(\operatorname{sov}(E \backslash \bar{S}(i)))) \\
& =\sum_{\substack{E \subseteq N \\
i \in E}} \omega_{|E|}(v(\bar{S}(\operatorname{sov}(E)))-v(\bar{S}(\operatorname{sov}(E)) \backslash \bar{S}(i))) .
\end{aligned}
$$

Since $m^{\omega}(N, S, v)$ need not be budget neutral, we normalize the weighted average marginal contributions of the players so that we obtain a budget neutral wage function that distributes the total production output value proportional to the weighted average marginal contributions of the employees. Following van der Laan and van den Brink (1998), we assign to every player a share $\frac{m_{i}^{\omega}(N, S, v)}{\sum_{j \in N} m_{j}^{\omega}(N, S, v)}$ in the production output value, and obtain the corresponding budget neutral wage function $\varphi^{\omega}$ that is given by

$$
\varphi_{i}^{\omega}(N, S, v)=\frac{m_{i}^{\omega}(N, S, v)}{\sum_{j \in N} m_{j}^{\omega}(N, S, v)} \cdot v\left(W_{S}\right) \quad \text { for all } i \in N .
$$

We call these weighted average marginal contributions (or shortly WAMC) wage functions. (Note that the productive fully employed firm condition implies that $v\left(W_{S}\right)$ and $\sum_{j \in N} m_{j}^{\omega}(N, S, v)$ are both positive. $)$ As mentioned above, the weights express the importance of the coalitions of different sizes in determining wages. The extreme case where we just take the average marginal contribution, i.e. taking weights $\omega_{k}^{B}=\frac{1}{2^{|N|-1}}$ for all $k \in\{1, \ldots,|N|\}$, yields the

\footnotetext{
6 In van den Brink et al. (2007) these weights are related to the cultural dimension of collectivism-individualism as considered in Hofstede (1980). In van den Brink and Ruys (2005) this model is used to endogenously determine firm size.

7 Weber (1988) treats these as solutions for TU-games and calls them symmetric probabilistic values.
} 
normalized Banzhaf value of the modified production game which is axiomatically characterized in van den Brink and van der Laan (1998) in a game theoretic context. ${ }^{8}$ The unique wage function for which the marginal contributions themselves are budget neutral, and thus need not be normalized, are the weights $\omega_{k}^{S h}=\frac{(|N|-k) !(k-1) !}{|N| !}$ for all $k \in\{1, \ldots,|N|\}$, that yield wages based on the Shapley value of the modified game. ${ }^{9}$

\section{Comparing wages between different hierarchical levels}

The main purpose of this paper is to compare the wage of a manager in a hierarchical production game with the wages of its successors. We assume any weight system $\omega=\left(\omega_{1}, \ldots, \omega_{|N|}\right)$ with $\omega_{k}>0, k \in\{1, \ldots,|N|\}$, to be given. In the literature, it is often argued that the wage of a manager in a firm is at least as high as the wage of any of its subordinates, see e.g., Simon (1957), Williamson (1967), Calvo and Wellisz $(1978,1979)$, and Carlson (1982). This property, which we refer to as vertical monotonicity, is satisfied by all WAMC-wage functions in case the production game is monotone. Moreover, if the production game is also supermodular, then according to any WAMC-wage function $\varphi^{\omega}$ the wage of a manager does not exceed the sum of the wages of its successors.

Theorem 4.1 Consider a hierarchical production game $(N, S, v)$ and manager $i \in M_{S}$.

(i) If $v$ is monotone then $\varphi_{i}^{\omega}(N, S, v) \geq \varphi_{j}^{\omega}(N, S, v)$ for all $j \in S(i)$;

(ii) If $v$ is monotone and supermodular then $\varphi_{i}^{\omega}(N, S, v) \leq \sum_{j \in S(i)} \varphi_{j}^{\omega}(N, S, v)$.

The proof of this theorem can be found in Appendix A. ${ }^{10}$ If firm $(N, S, v)$ has constant span of control, meaning that every manager has the same number of successors $s$, then Theorem 4.1 implies that the ratio between the wage of a manager and the average wage of its successors (if positive) lies between one and the span of control $s$. If moreover, the workers in $W_{S}$ are identical, meaning that $v(E)=v(F)$ for all $E, F \subseteq W_{S}$ with $|E|=|F|$, Theorem 4.1 yields the following corollary.

\footnotetext{
8 This is the normalization of the nonefficient Banzhaf value as considered in e.g., Owen (1975) and Dubey and Shapley (1979) and is a generalization to TU-games of the Banzhaf index introduced in Banzhaf (1965) as a power index for voting games.

9 The Shapley value (Shapley 1953) is one of the most applied solutions for TU-games. In firm models it has been applied in, e.g. Hart and Moore (1990) and Rajan and Zingales (1998). Taking the Shapley value of the modified game as wage function is a special case of the conjunctive and disjunctive permission value for games with a permission structure as characterized in van den Brink and Gilles (1996) and van den Brink (1997), respectively.

10 As follows from this proof, for the first inequality to hold we do not need the assumption that only workers that have no successors are productive. The second inequality needs this assumption. However, allowing productive workers in all levels we can prove in a similar way that $\varphi_{i}^{\omega}(N, S, v) \leq$ $\sum_{j \in S(i)} \varphi_{j}^{\omega}(N, S, v)$ whenever $i \in M_{S}$ is a null player in the production game $v$ defined on $2^{N}$.
} 
Corollary 4.2 For every hierarchical production game $(N, S, v)$ with monotone and supermodular $v$, identical workers and constant span of control $s$, it holds that $1 \leq \frac{\varphi_{i}^{\omega}(N, S, v)}{\varphi_{j}^{\omega}(N, S, v)} \leq s$ for all $i \in M_{S}$ and $j \in S(i)$ with $\varphi_{j}^{\omega}(N, S, v)>0$.

The bounds derived in this corollary are often assumed in the literature, see e.g. Williamson (1967). Next we argue that the bounds for the wage ratios given in Theorem 4.1 are sharp in the sense that there are hierarchical production games such that the inequalities are equalities. We first characterize the following types of subordinates of a manager. Successor $j$ of manager $i$ is indispensable for $i$ if every worker $h$ that is coordinated by $i$, but not by $j$, does not increase the productivity of any set of workers that does not contain workers coordinated by $j$ (or $j$ if $j$ itself is a worker). In other words, the workers that are coordinated by $i$ but not by $j$ need workers that are coordinated by $j$ (or $j$ itself) in order to be productive.

Second, worker $h$ is a dummy worker if it increases the productivity of every set of workers by the value $v(\{h\})$ which it also can produce on its own. In other words, there are no synergies when $h$ cooperates with other workers.

Definition 4.3 In the hierarchical production game $(N, S, v)$

(i) $j \in S(i)$ is indispensable for $i \in M_{S}$ if $v(E)=v(E \backslash\{h\})$ for every $h \in \bar{S}(i) \backslash \bar{S}(j)$ and every $E \subset W_{S}$ with $E \cap \bar{S}(j)=\emptyset$;

(ii) $h \in W_{S}$ is a dummy worker if $v(E)=v(E \backslash\{h\})+v(\{h\})$ for every $E \subseteq W_{S}$ with $h \in E$.

We characterize hierarchical production games for which the bounds of Theorem 4.1 are reached using indispensable and dummy workers. It turns out that a manager earns the same wage as one of its successors if this successor is indispensable for the manager. A manager earns the sum of the wages of its successors if all its subordinate workers are dummy workers.

Theorem 4.4 Consider a hierarchical production game $(N, S, v)$ and manager $i \in M_{S}$.

(i) If $j \in S(i)$ is indispensable for $i$ in $(N, S, v)$ then $\varphi_{i}^{\omega}(N, S, v)=\varphi_{j}^{\omega}(N, S, v)$;

(ii) If every $h \in \bar{S}(i)$ is a dummy worker in $(N, S, v)$ then $\varphi_{i}^{\omega}(N, S, v)=$ $\sum_{j \in S(i)} \varphi_{j}^{\omega}(N, S, v)$.

Again, the proof can be found in Appendix A. ${ }^{11}$ A class of production functions that gained a lot of attention in the literature is the class of constant elasticity of substitution production functions. A production function with

11 Redefining indispensability by saying that successor $j$ of manager $i$ is indispenable for $i$ if every employee $h$ that is coordinated by $i$ and not by $j$ does not increase the production value of any set of employees that does not contain $j$ and employees coordinated by $j$, the first statement also holds for firms where production takes place at any level (and not necessarily only on the bottom level with no successors). Redefining dummy workers by saying that employee $h$ is a dummy employee if $h$ and all its subordinates $j \in \widehat{S}(h)$ increase the productivity of every set of employees by the value which they also can produce on their own, the second statement also holds for firms where production takes place at any level. 
$n$ inputs $f: \mathbb{R}_{+}^{n} \rightarrow \mathbb{R}_{+}$is a supermodular CES production function if there exists $\rho \in(0,1], \gamma>0$ and $\alpha_{1}, \ldots, \alpha_{n}>0$ with $\sum_{i=1}^{n} \alpha_{i}=1$ such that $f(x)=$ $\gamma\left(\sum_{i=1}^{n} \alpha_{i}\left(x_{i}\right)^{\rho}\right)^{\frac{1}{\rho}}$. The parameter $\gamma$ is a scale parameter and $\rho$ is a parameter expressing the substitutability of the inputs. We need $\rho>0$ to obtain a supermodular production function. In our model the $n$ inputs are the $\left|W_{S}\right|$ labor inputs. Then $x_{i} \geq 0$ is the amount of labor that is provided by worker $i \in W_{S}$. Since we assume that workers can be either fully active or inactive, we represent the situation in which exactly the workers in the set $E \subseteq W_{S}$ are active by the labor input vector $x^{E} \in\{0,1\}^{\left|W_{S}\right|}$ given by $x_{i}^{E}=1$ if $i \in E$, and $x_{i}^{E}=0$ otherwise. Assuming identical workers (i.e. $\alpha_{i}=\frac{1}{n}, i \in\{1, \ldots, n\}$ ) and constant span of control, the CES production game then can be written as $v_{\rho}(E)=\gamma\left(\frac{|E|}{\left|W_{S}\right|}\right)^{\frac{1}{\rho}}$. For a hierarchical supermodular CES production game with constant span of control and identical workers the results in this section imply that the ratio between the wage of a manager and the wage of each of its successors is equal to the span of control $s$ in case we have a linear production game with substitutable labor inputs ${ }^{12}$ (i.e. $\rho=1$ ), while it is equal to one in case the production game is a Cobb-Douglas production game with indispensable labor inputs (i.e. $\rho \rightarrow 0)$.

\section{Properties of WAMC wage functions}

In this section, we discuss some properties of WAMC wage functions. It turns out that, besides the earlier mentioned properties of budget neutrality and vertical monotonicity [see Theorem 4.1.(i)], all these wage functions satisfy the following properties. First, an employee $i$ is an inessential employee in hierarchical production game $(N, S, v)$ if every worker that is coordinated by $i$, or $i$ itself if it is a worker, does not add anything in the production process, i.e. if $v(E)=v(E \backslash\{j\})$ for every $j \in \bar{S}(i)$ and $E \subseteq W_{S}$. The inessential employee property states that inessential employees are assigned a wage equal to zero. Second, a worker $i$ is a necessary worker if, without its labor effort nothing can be produced, i.e. if $v(E)=0$ for all $E \subseteq W_{S} \backslash\{i\}$. The necessary worker property states that necessary workers always earn at least as much as any other employee in the firm in case the production game is monotone. Every WAMC-wage function is characterized by the above mentioned four properties together with a weighted additivity which states how payoffs are determined if we add production games in the same firm structure. ${ }^{13}$ Since the weighted marginal contribution vectors corresponding to the Shapley weights are budget neutral by themselves, the Shapley wage function $\varphi^{S h}=\varphi^{\omega^{S h}}$ is additive meaning that for two production games $v, z$ and firm structure $S$ it

\footnotetext{
12 Note that, e.g., Williamson (1967) and Rajan and Zingales (2001) only consider linear production technologies and therefore cannot study the effect of labor substitutability on wages.

13 General weighted additivity properties of share functions for TU-games are considered in van der Laan and van den Brink (1998).
} 
holds that $\varphi^{S h}(N, S, v+z)=\varphi^{S h}(N, S, v)+\varphi^{S h}(N, S, z)$, where production game $(v+z): 2^{W_{S}} \rightarrow \mathbb{R}_{+}$is given by $(v+z)(E)=v(E)+z(E)$ for all $E \subseteq W_{S}$. For the other WAMC-wage functions the marginal contributions need to be normalized by the factor $\frac{v\left(W_{S}\right)}{\sum_{j \in N} m_{j}^{\omega}(N, S, v)}$. The reciprocal of this factor somehow measures how much the average weighted marginal contributions yield an excess (or deficit) on the budget. Since we only consider budget neutral wage functions, when adding two production games it seems reasonable to use these factors in determining the wages from those in the separate production games. Given some positive weight system $\omega=\left(\omega_{1}, \ldots, \omega_{|N|}\right)$, the corresponding WAMC-wage function $\varphi^{\omega}$ satisfies $\omega$-additivity meaning that for every pair of production games $v, z$ and firm structure $S$ it holds that $\frac{\sum_{j \in N} m_{j}^{\omega}(N, S, v+z)}{(v+z)\left(W_{S}\right)} \varphi(N, S, v+z)=$ $\frac{\sum_{j \in N} m_{j}^{\omega}(N, S, v)}{v\left(W_{S}\right)} \varphi(N, S, v)+\frac{\sum_{j \in N} m_{j}^{\omega}(N, S, z)}{z\left(W_{S}\right)} \varphi(N, S, z)$.

Theorem 5.1 A wage function satisfies budget neutrality, vertical monotonicity, the inessential employee property, the necessary worker property and $\omega$-additivity if and only if it equals $\varphi^{\omega}$.

The proof of this theorem can be found in Appendix B which also shows logical independence of the properties. An additional property that is relevant for analyzing remuneration in firms is self-duality of a wage function meaning that the wages in a hierarchical production game $(N, S, v)$ are the same if, instead of the modified game $r_{S, v}$ we consider its dual $r_{S, v}^{*}: 2^{N} \rightarrow \mathbb{R}_{+}$which assigns to every subset of employees the production output value that is lost by the fully employed firm if the employees in $E$ leave the firm, i.e. $r_{S, v}^{*}(E)=$ $r_{S, v}(N)-r_{S, v}(N \backslash E)$ for all $E \subseteq N$. Although we measured the importance of firm employees in the production of the firm by considering their contributions to production, the importance of firm employees often is measured in terms of what happens with production if employees leave the firm or stop being active. We call a WAMC-wage function $\varphi^{\omega}$ self-dual if $\varphi^{\omega}(N, S, v)=\varphi^{\omega^{*}}(N, S, v)$ where $\varphi_{i}^{\omega^{*}}(N, S, v)=\frac{m_{i}^{\omega^{*}}(N, S, v)}{\sum_{j \in N} m_{j}^{\omega^{*}}(N, S, v)} \cdot v\left(W_{S}\right)$, with $m_{i}^{\omega^{*}}(N, S, v)=\sum_{E \subset N} \omega_{|E|}\left(r_{S, v}^{*}(E)-\right.$ $\left.r_{S, v}^{*}(E \backslash\{i\})\right)$ for every $i \in N$ and hierarchical production game $(N, S, v)$. For selfdual wage functions it does not matter which of the two approaches we take.

Proposition 5.2 If the positive weight system $\omega=\left(\omega_{1}, \ldots, \omega_{|N|}\right)$ satisfies $\omega_{k}=$ $\omega_{|N|-k+1}$ for all $k \in\{1, \ldots,|N|\}$, then the corresponding WAMC-wage function $\varphi^{\omega}$ is self-dual.

The straightforward proof of this proposition also can be found in Appendix B. In particular the condition on the weight system in Proposition 5.2 holds for the Banzhaf- and Shapley wage functions ${ }^{14}$ mentioned at the end of Sect. 3. Moreover, given any WAMC-wage function $\varphi^{\omega}$, defining the weight system $\bar{\omega}=\left(\bar{\omega}_{1}, \ldots, \bar{\omega}_{|N|}\right)$ by $\bar{\omega}_{k}=\frac{1}{2}\left(\omega_{k}+\omega_{|N|-k+1}\right)$ yields a self-dual WAMC-wage function $\varphi^{\bar{\omega}}$.

${ }_{14}$ For the Shapley wage function this also follows directly from the dual game property of the Shapley value as shown by Kalai and Samet (1987). 


\section{An example: the Shapley wage function and constant elasticity of substitution production technologies}

In this section, we provide an illustration of a hierarchical production game and the results of the previous sections using the specific Shapley wage function (which is based on the Shapley value) and CES production functions.

Consider a two-level firm with three employees and firm structure $S$ on $N=\{1,2,3\}$ given by $S(1)=S(2)=\emptyset$ and $S(3)=\{1,2\}$ (see Fig. 2). Suppose that the firm produces an output with two identical labor inputs (that can be provided by the workers 1 and 2) according to a CES production function $f(x)=\gamma\left(\frac{1}{2}\left(\left(x_{1}\right)^{\rho}+\left(x_{2}\right)^{\rho}\right)\right)^{\frac{1}{\rho}}$, with $\gamma>0$ and $\rho \in(0,1]$. This yields the CES production game $v_{\rho}: 2^{W_{S}} \rightarrow \mathbb{R}_{+}$given by

$$
v_{\rho}(E)=\gamma\left(\frac{1}{2}|E|\right)^{\frac{1}{\rho}}=\left\{\begin{array}{cl}
0 & \text { if }|E|=0 \\
\gamma\left(\frac{1}{2}\right)^{\frac{1}{\rho}} & \text { if }|E|=1 \\
\gamma & \text { if }|E|=2 .
\end{array}\right.
$$

The wages according to the Shapley wage function (with $\omega_{1}^{S h}=\omega_{2}^{S h}=\frac{1}{2}$ ) for the manager 3 and the workers 1 and 2 are

$$
\varphi_{3}^{S h}\left(N, S, v_{\rho}\right)=\frac{\gamma}{3}\left(1+\left(\frac{1}{2}\right)^{\frac{1}{\rho}}\right) \quad \text { and } \quad \varphi_{i}^{S h}\left(N, S, v_{\rho}\right)=\frac{\gamma}{3}\left(1-\left(\frac{1}{2}\right)^{\frac{1}{\rho}+1}\right)
$$

for $i \in\{1,2\}$.

The ratio between the wages of manager 3 and workers 1 and 2 , denoted by $\delta(\rho)$, then is given by

$$
\delta(\rho)=\frac{\varphi_{3}^{S h}\left(N, S, v_{\rho}\right)}{\varphi_{1}^{S h}\left(N, S, v_{\rho}\right)}=\frac{1+(1 / 2)^{\frac{1}{\rho}}}{1-(1 / 2)^{\frac{1}{\rho}+1}} \quad \text { for } \rho \in(0,1]
$$

Note that $\delta(\rho)$ does not depend on the scale parameter $\gamma$. Since $\frac{d \delta(\rho)}{d \rho}=$ $-\frac{3 \ln (1 / 2)(1 / 2)^{\frac{1}{\rho}+1}}{\left(\rho\left(1-(1 / 2)^{\frac{1}{\rho}+1}\right)\right)^{2}}>0$ for $\rho \in(0,1]$, this ratio is increasing in $\rho$, i.e. the difference between the wages of the manager and the workers increases if the substitutability of the labor inputs increases.

If $\rho=1$ then production takes place according to a linear production technology with substitutable labor inputs and production game $v_{1}(E)=\frac{\gamma}{2}(|E|)$. In this case, the workers 1 and 2 are both dummy workers. The wages of the

Fig. 2 Firm structure $S$ of a two-level firm with $s=2$

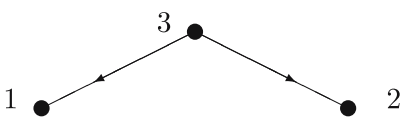


employees are $\varphi_{3}^{S h}\left(N, S, v_{1}\right)=\frac{\gamma}{2}$ and $\varphi_{i}^{S h}\left(N, S, v_{1}\right)=\frac{\gamma}{4}$ for $i \in\{1,2\}$, and thus the ratio $\delta(1)$ is equal to the span of control $s=2$.

If $\rho \rightarrow 0$ then the production game approaches a Cobb-Douglas production game given by $v_{0}(E)=\gamma x_{1}^{E} x_{2}^{E}$, and thus is given by $v_{0}(E)=\gamma$ if $E=W_{S}$, and $v_{0}(E)=0$ otherwise. Both labor inputs are indispensable in order to produce a positive production output value. In this case, the wages are equal and given by $\varphi_{i}^{S h}\left(N, S, v_{0}\right)=\frac{\gamma}{3}$ for $i \in\{1,2,3\}$, and thus $\delta(0)=1$.

For the intermediate case with $\rho=\frac{1}{2}$ the production game is given by $v_{1 / 2}(E)=\frac{\gamma}{4}(|E|)^{2}$. In this case the wages are $\varphi_{3}^{S h}\left(N, S, v_{1 / 2}\right)=\frac{5}{12}$ and $\varphi_{i}^{S h}\left(N, S, v_{1 / 2}\right)=\frac{7}{24}$ for $i \in\{1,2\}$, and thus $\delta\left(\frac{1}{2}\right)=\frac{10}{7}$. So, $1<\delta\left(\frac{1}{2}\right)<s$.

\section{Concluding remarks}

The purpose of this paper is to gain insight in the internal wage distribution in a hierarchically structured firm of given size. Although most literature on the firm uses noncooperative game theory (which is suitable to analyze situations where binding agreements are not possible), we used a cooperative game theoretic model in which coalitions or teams of workers can generate a production output value when they are coordinated by their managers and agree to perform the task assigned to their position.

It is our belief that for understanding firms as production organizations, eventually concepts from both cooperative and noncooperative decision making are needed. There is a large literature on noncooperative models of firms using, for example, principal-agent theory to explain issues of moral hazard (see, e.g., Holmström 1979 and Grossman and Hart 1983). With the model presented in this paper we try to complement this literature with a cooperative model. We showed that the wage functions derived in this way have properties that fit with assumptions made in the literature on the firm. Although the main attention in the Theory of the Firm is still on noncooperative models, some interesting other attempts have been made to incorporate cooperative behavior in understanding firms, such as the literature on incomplete contracts and collective binary decision making. The literature on incomplete contracts tries to explain the distribution of residual rights concerning the control over noncontractable assets (see, e.g., Grossman and Hart 1986; Hart and Moore 1990, 1999; Maskin and Tirole 1999). While in this literature the ownership of assets is central, in the underlying paper it is not ownership but, as formulated by Rajan and Zingales (1998), the control of access to a productive asset which is the issue. When a worker occupies a position in a firm this means that he gets access to the production facilities. In return for his wage, the worker offers his labor input which generates the production output value when operating the production facility. When a manager occupies a position then he gets (partial) control over the access of his subordinates to the productive asset. He earns his compensation by using this control in a way to facilitate his subordinate workers so that they can generate an output value. 
In the hierarchical production game of this paper, a worker needs permission from all its superiors to be active in the production process. This is closely related to hierarchies in the sense of, e.g. Sah and Stiglitz (1986), Ben-Yashar and Nitzan (2001) and Koh (2005), who consider a collective decision maker that has to choose whether to accept or reject a project proposal. In a hierarchy a project proposal is accepted if and only if all individuals accept. Instead of evaluating the performance of the firm as a collective decision maker, in this paper we focussed on the distribution of the given earnings of the firm over the individual employees. As an alternative to a hierarchy, Sah and Stiglitz (1986) also consider a polyarchy in which a project proposal is accepted if and only if at least one individual accepts. To study polyarchies we need to apply the more general games with (acyclic) permission structures being games in which the players in a cooperative TU-game are part of a hierarchical (permission) structure such that players need permission from their predecessors to cooperate with other players. In these acyclic permission structures every player can have more than one predecessor. Two approaches are distinguished. In the conjunctive approach as developed in Gilles et al. (1992) and van den Brink and Gilles (1996), players need permission from all their predecessors in order to cooperate. On the other hand, in the disjunctive approach as developed in Gilles and Owen (1994) and van den Brink (1997) every player (except the top-player) needs permission from at least one of its predecessors. In this sense a polyarchy as a collective decision making organization is closely related to the disjunctive approach, while a hierarchy is closely related to the conjunctive approach. Clearly, for permission tree structures as considered in this paper these two approaches are the same.

Another special class of games with a permission structure in which the permission structure is a tree are the peer group games as introduced in Brânzei et al. (2002). Instead of considering the (production) game to be defined on the set of players that have no successors, in peer group games the original game is defined on the set of all players, but it is an additive game, i.e. every coalition can generate a worth equal to the sum of the worths that the members of the coalition can earn on their own. For the purpose of the underlying paper peer group games are not suitable since by additive games we can only model linear production firms, and thus differences in labor substitutability cannot be studied by that model. Studying the effect of such differences has been the main topic of this paper.

Acknowledgments I would like to thank Pieter Ruys and two anonymous referees for useful remarks on a previous draft of the paper. Financial support from the Netherlands organization for scientific research (NWO) ESR-grant 510-01-0504 is gratefully acknowledged.

\section{Appendix A: Proofs of Sect. 4}

In this appendix, we give the proofs of the main theorems of the paper stated in Sect. 4. We use the following results for hierarchical production games $(N, S, v)$ which follow from Gilles et al. (1992): 
(i) If $v$ is monotone then $r_{S, v}$ is monotone, i.e. $r_{S, v}(E) \leq r_{S, v}(F)$ for all $E \subseteq F \subseteq N$

(ii) If $v$ is monotone and supermodular then $r_{S, v}$ is supermodular, i.e. $r_{S, v}(E)+$ $r_{S, v}(F) \leq r_{S, v}(E \cup F)+r_{S, v}(E \cap F)$ for all $E, F \subseteq N$.

\section{Proof of Theorem 4.1}

Let $(N, S, v)$ be a hierarchical production game.

(i) For every $i \in M_{S}$ and $j \in S(i)$, monotonicity of $r_{S, v}$ implies the following facts (which follow from a more general result in van den Brink and Gilles 1996):

(a) Since $\operatorname{sov}(E)=\operatorname{sov}(E \backslash\{j\})$ for all $E \subset N$ with $i \notin E$, it holds that $r_{S, v}(E)-r_{S, v}(E \backslash\{j\})=0$ for all $E \subset N$ with $i \notin E, j \in E$;

(b) Since $\operatorname{sov}(E \backslash\{i\}) \subseteq \operatorname{sov}(E)$ for all $E \subseteq N$, it holds that $r_{S, v}(E)-r_{S, v}(E \backslash\{i\}) \geq$ 0 for all $E \subset N$ with $i \in E, j \notin E$;

(c) Since $\operatorname{sov}(E \backslash\{i\}) \subseteq \operatorname{sov}(E \backslash\{j\})$ for all $E \subseteq N$, it holds that $r_{S, v}(E)-r_{S, v}(E \backslash$ $\{j\}) \leq r_{S, v}(E)-r_{S, v}(E \backslash\{i\})$ for all $E \subseteq N$ with $i, j \in E$.

With this it follows that

$$
\begin{aligned}
m_{i}^{\omega}(N, S, v)= & \sum_{\substack{E \subseteq N \\
i \in E}} \omega_{|E|}\left(r_{S, v}(E)-r_{S, v}(E \backslash\{i\})\right) \\
= & \left(\sum_{\substack{E \subseteq N \\
i, j \in E}} \omega_{|E|}\left(r_{S, v}(E)-r_{S, v}(E \backslash\{i\})\right)\right) \\
& +\left(\sum_{\substack{E \subset N \\
i \in E, j \notin E}} \omega_{|E|}\left(r_{S, v}(E)-r_{S, v}(E \backslash\{i\})\right)\right) \\
\geq & \sum_{\substack{E \subseteq N \\
i, j \in E}} \omega_{|E|}\left(r_{S, v}(E)-r_{S, v}(E \backslash\{j\})\right) \\
= & \sum_{\substack{E \subseteq N \\
j \in E}} \omega_{|E|}\left(r_{S, v}(E)-r_{S, v}(E \backslash\{j\})\right)=m_{j}^{\omega}(N, S, v) .
\end{aligned}
$$

Since $\varphi^{\omega}(N, S, v)$ is obtained by normalizing these marginal contributions according to (2), it follows that $\varphi_{i}^{\omega}(N, S, v) \geq \varphi_{j}^{\omega}(N, S, v)$.

(ii) By monotonicity and supermodularity of $r_{S, v}$, for every $i \in M_{S}$ with $S(i)=\left\{j_{1}, \ldots, j_{s}\right\}$, we have for every $k \in\{1, \ldots, s\}$ and $E \subseteq N$ that

$$
r_{S, v}(E) \geq r_{S, v}\left(E \backslash\left\{j_{k}\right\}\right)+r_{S, v}\left(E \backslash \bigcup_{l=k+1}^{s}\left\{j_{l}\right\}\right)-r_{S, v}\left(E \backslash \bigcup_{l=k}^{s}\left\{j_{l}\right\}\right)
$$


But then

$$
\begin{aligned}
(s-1) r_{S, v}(E) & =\sum_{k=1}^{s-1} r_{S, v}(E) \\
& \geq \sum_{k=1}^{s-1}\left(r_{S, v}\left(E \backslash\left\{j_{k}\right\}\right)+r_{S, v}\left(E \backslash \bigcup_{l=k+1}^{s}\left\{j_{l}\right\}\right)-r_{S, v}\left(E \backslash \bigcup_{l=k}^{s}\left\{j_{l}\right\}\right)\right) \\
& =\sum_{k=1}^{s-1}\left(r_{S, v}\left(E \backslash\left\{j_{k}\right\}\right)\right)+r_{S, v}\left(E \backslash\left\{j_{s}\right\}\right)-r_{S, v}\left(E \backslash \bigcup_{l=1}^{s}\left\{j_{l}\right\}\right) \\
& =\sum_{k=1}^{s} r_{S, v}\left(E \backslash\left\{j_{k}\right\}\right)-r_{S, v}\left(E \backslash \bigcup_{l=1}^{s}\left\{j_{l}\right\}\right) \\
& =\sum_{j \in S(i)} r_{S, v}(E \backslash\{j\})-r_{S, v}(E \backslash S(i)) .
\end{aligned}
$$

Since all players in $M_{S}$ are null players in $\bar{v}$, it holds that $r_{S, v}(E \backslash\{i\})=r_{S, v}(E \backslash S(i))$ for every $i \in M_{S}$, and thus $(s-1) r_{S, v}(E) \geq \sum_{j \in S(i)} r_{S, v}(E \backslash\{j\})-r_{S, v}(E \backslash\{i\})$, which is equivalent to

$r_{S, v}(E)-r_{S, v}(E \backslash\{i\}) \leq s r_{S, v}(E)-\sum_{j \in S(i)} r_{S, v}(E \backslash\{j\})=\sum_{j \in S(i)}\left(r_{S, v}(E)-r_{S, v}(E \backslash\{j\})\right)$.

With (1) it follows that $m_{i}^{\omega}(N, S, v) \leq \sum_{j \in S(i)} m_{j}^{\omega}(N, S, v)$, and thus with (2), it follows that $\varphi_{i}^{\omega}(N, S, v) \leq \sum_{j \in S(i)} \varphi_{j}^{\omega}(N, S, v)$.

\section{Proof of Theorem 4.4}

Let $(N, S, v)$ be a hierarchical production game and let $i \in M_{S}$.

(i) Let $j \in S(i)$ be indispensable for $i \in M_{S}$. Then

(a) $r_{S, v}(E)-r_{S, v}(E \backslash\{i\})=0$ if $i \in E, j \notin E$;

(b) $r_{S, v}(E)-r_{S, v}(E \backslash\{j\})=0$ if $i \notin E, j \in E$;

(c) $r_{S, v}(E)=r_{S, v}(E \backslash \bar{S}(i))$ for all $E \subset N$ with $E \cap \bar{S}(j)=\emptyset$.

From this and the facts that $r_{S, v}(E \backslash\{i\})=r_{S, v}(E \backslash \bar{S}(i)), r_{S, v}(E \backslash\{j\})=r_{S, v}(E \backslash \bar{S}(j))$ for all $E \subseteq N$, and $\bar{S}(j) \subset \bar{S}(i)$ it follows that

$$
\begin{aligned}
m_{i}^{\omega}(N, S, v) & =\sum_{\substack{E \subseteq N \\
i \in E}} \omega_{|E|}\left(r_{S, v}(E)-r_{S, v}(E \backslash\{i\})\right) \\
& =\sum_{\substack{E \subseteq N \\
i, j \in E}} \omega_{|E|}\left(r_{S, v}(E)-r_{S, v}(E \backslash\{i\})\right)=\sum_{\substack{E \subseteq N \\
i, j \in E}} \omega_{|E|}\left(r_{S, v}(E)-r_{S, v}(E \backslash \bar{S}(i))\right)
\end{aligned}
$$




$$
\begin{aligned}
= & \sum_{\substack{E \subseteq N \\
i, j \in E}} \omega_{|E|}\left(r_{S, v}(E)-r_{S, v}((E \backslash \bar{S}(j)) \backslash \bar{S}(i))\right. \\
= & \sum_{\substack{E \subseteq N \\
i, j \in E}} \omega_{|E|}\left(r_{S, v}(E)-r_{S, v}(E \backslash \bar{S}(j))\right) \\
= & \sum_{\substack{E \subseteq N \\
i, j \in E}} \omega_{|E|}\left(r_{S, v}(E)-r_{S, v}(E \backslash\{j\})\right) \\
= & \sum_{\substack{E \subseteq N \\
j \in E}} \omega_{|E|}\left(r_{S, v}(E)-r_{S, v}(E \backslash\{j\})\right)=m_{j}^{\omega}(N, S, v) .
\end{aligned}
$$

With (2) it then follows that $\varphi_{i}^{\omega}(N, S, v)=\varphi_{j}^{\omega}(N, S, v)$.

(ii) Suppose that all $h \in \bar{S}(i)$ are dummy workers. Then

(a) $r_{S, v}(E)-r_{S, v}(E \backslash\{i\})=\sum_{h \in \operatorname{sov}(E) \cap \bar{S}(i)} v(\{h\})$ for all $E \subseteq N$ with $i \in E$;

(b) $r_{S, v}(E)-r_{S, v}(E \backslash\{j\})=\sum_{h \in \operatorname{sov}(E) \cap \bar{S}(j)} v(\{h\})$ for all $j \in S(i) \cap E$;

(c) $\bar{S}(i) \cap \bar{S}(j)=\bar{S}(j)$ for all $j \in S(i)$;

(d) $r_{S, v}(E)-r_{S, v}(E \backslash\{j\})=0$ if $j \in S(i) \cap E, i \notin E$.

With this it follows that

$$
\begin{aligned}
m_{i}^{\omega}(N, S, v) & =\sum_{\substack{E \subseteq N \\
i \in E}} \omega_{|E|}\left(r_{S, v}(E)-r_{S, v}(E \backslash\{i\})\right) \\
& =\sum_{\substack{E \subseteq N \\
i \in E}} \omega_{|E|}\left(\sum_{h \in \operatorname{sov}(E) \cap \bar{S}(i)} v(\{h\})\right) \\
& =\sum_{E \subseteq N} \sum_{i \in E} \omega_{j \in S(i) \cap E}\left(\sum_{h \in \operatorname{sov}(E) \cap \bar{S}(i) \cap \bar{S}(j)} v(\{h\})\right) \\
& =\sum_{j \in S(i)} \sum_{\substack{E \subseteq N \\
i, j \in E}} \omega_{|E|}\left(\sum_{h \in \operatorname{sov}(E) \cap \bar{S}(j)} v(\{h\})\right) \\
& =\sum_{j \in S(i)} \sum_{\substack{E \subseteq N \\
i, j \in E}} \omega_{|E|}\left(r_{S, v}(E)-r_{S, v}(E \backslash\{j\})\right) \\
& =\sum_{j \in S(i)} \sum_{\substack{E \subseteq N \\
j \in E}} \omega_{|E|}\left(r_{S, v}(E)-r_{S, v}(E \backslash\{j\})\right)=\sum_{j \in S(i)} m_{j}^{\omega}(N, S, v) .
\end{aligned}
$$

With (2) it then follows that $\varphi_{i}^{\omega}(N, S, v)=\sum_{j \in S(i)} \varphi_{j}^{\omega}(N, S, v)$. 


\section{Appendix B: Proofs of Sect. 5}

In this appendix, we give the proofs of Sect. 5 and show logical independence of the properties of Theorem 5.1.

\section{Proof of Theorem 5.1}

It is straightforward and left to the reader to verify that the wage functions $\varphi^{\omega}$ satisfy these properties. To show uniqueness, ${ }^{15}$ suppose that $\varphi$ is a wage function that satisfies the five properties (with $\omega$-additivity for some positive weight system) and let $(N, S, v)$ be a hierarchical production game. To show that $\varphi(N, S, v)=\varphi^{\omega}(N, S, v)$, first consider for every $T \subseteq W_{S}, T \neq \emptyset$, and $\alpha>0$, the production game $v_{T, \alpha}: 2^{W_{S}} \rightarrow \mathbb{R}_{+}$given by $v_{T, \alpha}(E)=\alpha$ if $T \subseteq E$, and $v_{T, \alpha}(E)=0$ otherwise (in game theoretic terms such production games are positively scaled unanimity games). Let $I(T, S):=\{i \in N \mid T \cap(\{i\} \cup \widehat{S}(i))=\emptyset\}$ be the set of all inessential employees in $\left(N, S, v_{T, \alpha}\right)$. The necessary worker property implies that there exists a constant $c^{*} \in \mathbb{R}$ such that $\varphi_{i}\left(N, S, v_{T, \alpha}\right)=c^{*}$ for all $i \in W_{S} \backslash I(T, S)$, and $\varphi_{i}\left(N, S, v_{T, \alpha}\right) \leq c^{*}$ for all other employees. With vertical monotonicity it follows that $\varphi_{i}\left(N, S, v_{T, \alpha}\right) \geq c^{*}$ for all $i \in M_{S} \backslash I(T, S)$. Thus $\varphi_{i}\left(N, S, v_{T, \alpha}\right)=c^{*}$ for all $i \in N \backslash I(T, S)$. The inessential employee property implies that $\varphi_{i}\left(N, S, v_{T, \alpha}\right)=0$ for all $i \in I(T, S)$. Then, budget neutrality determines that $c^{*}=\frac{\alpha}{|N \backslash I(T, S)|}$. Thus $\varphi\left(N, S, v_{T, \alpha}\right)$ is uniquely determined in case $\alpha>0$.

If $\alpha<0$ then $v_{T,-\alpha}$ is a monotone supermodular production game. Since $v_{T, \alpha}(E)+v_{T,-\alpha}(E)=0$ for all $E \subseteq W_{S}$, it follows with $\omega$-additivity that also in this case $\varphi\left(N, S, v_{T, \alpha}\right)$ is uniquely determined.

Since for every production game $v: 2^{W_{S}} \rightarrow \mathbb{R}_{+}$the numbers $\alpha_{T}, T \subseteq W_{S}$, such that $v=\sum_{T \subseteq W_{S}, T \neq \emptyset} v_{T, \alpha_{T}}$ are unique (see Shapley 1953) and above we determined the values $\varphi\left(N, S, v_{T, \alpha_{T}}\right), \omega$-additivity of $\varphi$ implies that $\varphi(N, S, v)$ is uniquely determined. Thus, there can be at most one wage function that satisfies the five properties. Since the wage function $\varphi^{\omega}$ satisfies the five properties $\varphi$ must be equal to $\varphi^{\omega}$.

We show logical independence of the five properties used in characterizing the WAMC-wage functions $\varphi^{\omega}$ by the following alternative wage functions:

1. For $\alpha>0, \alpha \neq 1$, the wage function $\varphi^{\alpha \omega}$ satisfies the axioms of Theorem 5.1 except budget neutrality.

2. The wage function $\varphi$ that assigns to every hierarchically structured firm wages given by the Shapley value of the game $\bar{v}$, i.e. $S h_{i}(\bar{v})=\sum_{\substack{E \subseteq N \\ i \in E}} \omega_{|E|}$. $(\bar{v}(E)-\bar{v}(E \backslash\{i\}))$, satisfies the axioms of Theorem 5.1 except vertical monotonicity.

15 For the Shapley wage function this proof is implicitly given in van den Brink (1997). Here we generalize this proof along similar lines as the Shapley share function for TU-games is generalized in van der Laan and van den Brink (1998). 
3. The wage function $\varphi$ that distributes the total production value $v\left(W_{S}\right)$ equally over all employees (i.e. $\varphi_{i}(N, S, v)=\frac{v\left(W_{S}\right)}{|N|}$ for all $\left.i \in N\right)$ satisfies the axioms of Theorem 5.1 except the inessential employee property.

4. The wage function that assigns the total production output value to the top, i.e. $\varphi_{i_{0}}(N, S, v)=v\left(W_{S}\right)$, and $\varphi_{i}(N, S, v)=0$ for all $i \in N \backslash\left\{i_{0}\right\}$, satisfies the axioms of Theorem 5.1 except the necessary worker property.

5. Every WAMC-wage function $\varphi^{\bar{\omega}}, \bar{\omega} \neq \omega$, satisfies the axioms of Theorem 5.1 except $\omega$-additivity.

\section{Proof of Proposition 5.2}

This proposition follows since for every $E \subseteq N, i \in E$, we have that $\sum_{\substack{E \subseteq N \\ i \in E}} \omega_{|E|}$. $\left(r_{S, v}^{*}(E)-r_{S, v}^{*}(E \backslash\{i\})\right)=\sum_{\substack{E \subseteq N \\ i \in E}} \omega_{|E|}\left(r_{S, v}(N)-r_{S, v}(N \backslash E)-r_{S, v}(N)+r_{S, v}(N \backslash\right.$ $(E \backslash\{i\})))=\sum_{\substack{E \subseteq N \\ i \in E}} \omega_{|E|}\left(r_{S, v}(N \backslash(E \backslash\{i\}))-r_{S, v}(N \backslash E)\right)=\sum_{\substack{E \subseteq N \\ i \in E}} \omega_{|E|}\left(r_{S, v}((N \backslash\right.$ $\left.E) \cup\{i\}))-r_{S, v}(N \backslash E)\right)=\sum_{\substack{E \subseteq N \\ i \in E}} \omega_{|N|-|E|+1}\left(r_{S, v}(E)-r_{S, v}(E \backslash\{i\})\right)$.

\section{References}

Alchian AA, Demsetz H (1972) Production, information costs, and economic organization. Am Econ Rev 62:777-795

Banzhaf JF (1965) Weighted voting doesn’t work: a mathematical analysis. Rutgers Law Rev 19:317-343

Ben-Yashar R, Nitzan S (2001) The robustness of optimal organizational architectures: a Note on hierarchies and polyarchies. Soc Choice Welfare 18:155-163

Brânzei R, Fragnelli V, Tijs S (2002) Tree connected peer group situations and peer group games. Math Methods Oper Res 55:93-106

van den Brink R (1997) An axiomatization of the disjunctive permission value for games with a permission structure. Int J Game Theory 26:27-43

van den Brink R, Gilles RP (1996) Axiomatizations of the conjunctive permission value for games with Permission Structures. Games Econ Behav 12:113-126

van den Brink R, van der Laan G (1998) Axiomatizations of the normalized Banzhaf value and the Shapley value. Soc Choice Welfare 15:567-582

van den Brink R, Ruys PHM (2005) Positional wages, market wages and firm size. Tinbergen Discussion Paper 05/020-1, Tinbergen Institute and Free University, Amsterdam

van den Brink R, Ruys PHM, Semenov R (2007) Governance of clubs and firms with cultural dimensions. Mimeo, Tinbergen Institute and Free University, Amsterdam

Calvo GA, Wellisz S (1978) Supervision, loss of control, and the optimum size of the firm. J Pol Econ 86:943-952

Calvo GA, Wellisz S (1979) Hierarchy, ability and income distribution. J Pol Econ 87:991-1010

Carlson NW (1982) Time rates tighten their grip on manufacturing industries. Mon Labor Rev $15-22$

Dubey P, Shapley LS (1979) Mathematical properties of the Banzhaf power index. Math Oper Res 4:99-131

Gilles RP, Owen G (1994) Games with permission structures: The disjunctive approach. Mimeo, Department of Economics, Virginia Polytechnic Institute and State University, Blacksburg, Virginia

Gilles RP, Owen G, van den Brink R (1992) Games with permission structures: the conjunctive approach. Int J Game Theory 20:277-293

Grossman SJ, Hart OD (1983) An analysis of the principal-agent problem. Econometrica 51:7-45 
Grossman SJ, Hart OD (1986) The costs and benefits of ownership: a theory of vertical and lateral integration. J Pol Econ 94:691-719

Hart O, Moore J (1990) Property rights and the nature of the firm. J Polit Econ 98:1119-1158

Hart O, Moore J (1999) Foundations of incomplete contracts. Rev Econ Stud 66:115-138

Hofstede G (1980) Culture's consequences. International differences in work-related values. Sage Publications, Beverly Hills

Holmström B (1979) Moral hazard and observability. Bell J Econ 10:74-91

Ichiishi T (1993) The cooperative nature of the firm. Cambridge University Press, Cambridge

Kalai E, Samet D (1987) On weighted Shapley values. Int J Game Theory 16:205-222

Keren M, Levhari D (1979) The optimum span of control in a pure hierarchy. Manage Sci 25:11621172

Keren M, Levhari D (1983) The internal organization of the firm and the shape of average costs. Bell J Econ 14:474-486

Koh WTH (2005) Optimal sequential decision architectures and the robustness of hierarchies and polyarchies. Soc Choice Welfare 24:397-411

van der Laan G, van den Brink R (1998) An axiomated class of share functions for $N$-person games. Theory Decision 44:117-148

Maskin E, Tirole J (1999) Unforeseen contingencies and incomplete contracts. Rev Econ Stud 66:83-114

Milgrom P, Roberts J (1994) The economics of modern manufacturing: technology, strategy and organization. Am Econ Rev 80:511-528

Owen G (1975) Multilinear extensions and the Banzhaf value. Nav Res Logis Q 22:741-750

Radner R (1992) Hierarchy: the economics of managing. J Econ Lit 30:1382-1415

Rajan RG, Zingales L (1998) Power in a theory of the firm. Q J Econ 113:387-432

Rajan RG, Zingales L (2001) The firm as a dedicated hierarchy: a Theory of the origins and growth of firms Q J Econ 116:805-851

Sah RK, Stiglitz JE (1986) The architecture of economic systems: hierarchies and polyarchies. Am Econ Rev 76:716-727

Shapley LS (1953) A value for $n$-person games. In: Kuhn HW, Tucker AW (eds) Ann Math Stud 28 (Contributions to the theory of games Vol. 2) pp 307-317. Princeton University Press, Princeton

Simon HA (1957) The compensation of executives. Sociometry 20:32-35

Weber RJ (1988) Probabilistic values for Games. In: Roth AE (ed) The Shapley value: Essays in Honor of Lloyd S. Shapley, Cambridge University Press, Cambridge

Williamson OE (1967) Hierarchical control and optimum firm size. J Pol Econ 75:123-138 